\title{
Maternal Effects in Ontogeny and Evolution
}

\author{
Maternal Effects in Mammals. Edited by Dario Maestripieri and Jill M. Mateo. \\ Chicago: University of Chicago Press. 2009. 360 pp., \$90.00 (cloth). \\ ISBN 978-0-226-50119-2
}

\author{
Lynn A. Fairbanks
}

Published online: 27 November 2009

(C) The Author(s) 2009. This article is published with open access at Springerlink.com

Maternal Effects in Mammals is an authoritative volume that provides a comprehensive review of new developments on a topic of importance in evolutionary biology, developmental psychology, and biomedical research. Maternal effects are defined here as effects of the mother's phenotype on offspring phenotype, separate from the direct effects of genes inherited from the mother. It has been a decade since the publication of Maternal Effects as Adaptations (Mousseau and Fox 1998) brought this topic to the forefront in population biology. This edited volume builds on that earlier work, with a focus on mammals. The features that define the mammalian order, internal gestation, lactation and maternal care, make mammalian mothers particularly good candidates for exhibiting maternal effects on the health and fitness of their offspring. For many mammalian species, these early influences are augmented by an extended period of immaturity and continuing relationships between mothers and offspring into adulthood. As the chapters in this volume demonstrate, maternal effects can be a powerful force influencing individual development and the evolution of populations.

The book is composed of 14 chapters that cover the theoretical basis in population biology, review the wealth of new data demonstrating maternal effects in different mammalian groups, and provide evidence for some of the proximate mechanisms that mediate maternal effects on offspring phenotypes. It is noteworthy that a large percent-

\footnotetext{
L. A. Fairbanks $(\square)$

Department of Psychiatry and Biobehavioral Sciences,

University of California at Los Angeles,

760 Westwood Plaza,

Los Angeles, CA 90095, USA

e-mail: LFairbanks@mednet.ucla.edu
}

age of the material referenced here has appeared in the last decade, and includes the use of a number of new molecular genetics technologies. This volume also expands the topic of maternal effects into new physiological, social, and behavioral domains.

After a brief introduction by the editors, the book begins with two chapters that present the theoretical background for maternal effects using quantitative evolutionary biological models. Cheverud and Wolf illustrate the complexity of maternal effects in a model that includes components for genetic and environmental influences on the mother's phenotypes, direct genetic and environmental effects on offspring phenotypes, and a possibility of interactions of maternal effects with offspring genotype and with current environment. The resulting model predicts that maternal effects can accelerate, slow, and even reverse the direction of selection on offspring traits alone, depending on the direction and degree of genetic covariance between maternal and offspring effects. In Chapter 3, Wade, Priest, and Cruikshank present a fitness model of maternal effects that reflects the theory of Relaxed Selective Constraint. They then take an innovative approach to testing the theory using molecular genetic sequence data and gene expression profiles for a maternal effects gene in mice. An interesting feature of these analyses is that much of the data came from publicly available shared resources in GenBank. These two theoretical chapters provide information on quantitative approaches to evolutionary biology modeling, combined with the use of experimental methods and new genetic technologies to test predictions from the models. They also underscore the pitfalls of ignoring maternal effects if the goal is to understand population level evolutionary processes. This point is supported empirically in the chapter by McAdam that effectively documents the impact of maternal effects on population cycles in red squirrels. 
Most of the chapters that follow focus on demonstrating maternal effects in specific taxonomic groups. For example, Wilson and Festa-Bianchet (Chapter 5) and Bowen (Chapter 6) review the extensive body of information available on maternal effects in wild ungulates and pinnipeds. Because both ungulates and pinnipeds are relatively easy to follow and observe in the wild, studies have been able to provide consistent evidence for effects of mother's age, experience, and condition on offspring survival, birth weight, and early growth rate in different species. This research also suggests that both environmental and genetic maternal effects are involved in influencing offspring development, and that genetic maternal effects may be more evident under some environmental circumstances than others.

Several chapters in this volume take a more behavioral and physiological approach to maternal effects, with consideration of proximate mechanisms that mediate between maternal phenotypes and offspring outcomes. For example, Maestripieri expands the scope of maternal effects beyond growth and physical development in his chapter on maternal effects in primates. Research in captive and provisioned free-ranging populations has provided evidence of maternal effects on offspring social behavior, social preferences, reactivity to the environment, and intergenerational transmission of maternal behavior. Galef presents an interesting chapter on the many ways mammalian mothers influence the food choices and feeding behavior of their offspring. Mateo reviews studies of the effects of prenatal exposure to maternal hormones and the effects of postnatal physical environment and maternal care on offspring development across taxonomic groups. In an example from her own research, she describes the role of the mother in the behavioral and glucocorticoid response to predators in Belding's ground squirrels. Holekamp and Dloniak cover hormonal and behavioral effects in a review of maternal effects in fissiped carnivores, including data on the relationship between mother's dominance rank and androgen levels during pregnancy and the later sexual and aggressive behavior of juvenile offspring in wild spotted hyenas. An interesting twist on maternal gestational effects is provided by Vandenbergh in a chapter that describes the persistent effects of intrauterine position on development in rodents. Female fetuses located between two male fetuses are exposed to levels of androgens that can influence brain development and have lifelong effects on morphology, behavior, and reproduction. Unlike most other maternal effects described in this volume, however, intrauterine position may be better considered an effect of sibling phenotypes on individual development than of maternal phenotypes.

A noteworthy chapter by Champagne and Curley (Chapter 9) provides information on an epigenetic mecha- nism that can mediate maternal effects. Methylation of specific sites in promoter regions of genes is known to prevent RNA access and keep the methylated genes in an inactive state. Because DNA methylation patterns are maintained through cell division, they can result in persistent reductions in expression of the gene in that tissue. This process has now been shown to mediate effects of natural variation in maternal care on offspring behavior and stress reactivity in rats. Offspring that experienced a lower quality of maternal care had higher levels of DNA methylation and reduced gene expression in hippocampal glucocorticoid receptors. The importance of DNA methylation in this process was verified when treatment of the offspring with agents that promote demethylation reversed the differences produced by the lower quality care. This type of controlled experimental research provides a necessary supplement to the more naturalistic and uncontrolled results presented in other chapters.

The book ends with a discursive chapter by Bjorklund, Grotuss, and Csinady on the possible role of maternal effects in the evolution of human intelligence, followed by a summary of the book by the editors. Mateo and Maestripieri call for more interdisciplinary research with greater attention to research on proximate mechanisms that mediate maternal effects on offspring outcomes. They also suggest more longitudinal studies of wild populations using within female designs and consideration of possible sex differences in maternal effects on offspring development.

Together, these chapters support the importance of maternal effects on individual development and on evolutionary processes. Effects on offspring phenotypes are particularly strong during early development and tend to decline with offspring age and independence from the mother. For some outcomes, however, maternal effects can be measured in the next generation. At the level of the population, maternal effects can exceed direct genetic effects in explaining trait variance, and can exert strong effects on the pace and direction of selection.

One notable omission in this volume is a chapter on fetal programming theory. The theory and supporting data that maternal nutrition during gestation has lifelong consequences for offspring health through early programming of metabolic processes are only briefly mentioned here. Further consideration of this literature would have made the volume of greater interest to biomedical scientists studying metabolic disorders like obesity, hypertension, diabetes, and cardiovascular disease. Another weakness in many of the chapters is the tendency to label correlations between maternal and offspring phenotypes as maternal effects without acknowledging the need to control for direct genetic or environmental effects on offspring traits. 
Who should read this book? Most of the chapters are written at an advanced level and assume some prior knowledge of terminology and theory. As a result, this volume would be most appropriate for upper division students, graduate students, post docs, and practitioners in the field. This is a particularly important book to read for biomedical geneticists who are not, as yet, considering maternal effects in the search for genetic influences on human diseases and disorders. It would also be of value for developmental and biobehavioral psychologists interested in parental influences on offspring development. This book achieves the goal of synthesizing a large body of information, much of it produced since the last volume on this topic was published. It is likely to promote greater cross-disciplinary awareness, and stimulate further research in the field.

Open Access This article is distributed under the terms of the Creative Commons Attribution Noncommercial License which permits any noncommercial use, distribution, and reproduction in any medium, provided the original author(s) and source are credited.

\section{References}

Mousseau TA, Fox CW (eds) (1998) Maternal Effects as Adaptations. Oxford University Press, New York 